\title{
Culturas na rede: experiências de intercâmbio entre estudantes brasileiros, espanhóis e argentinos mediadas por Ambiente Virtual de Aprendizagem
}

\author{
Martha Kaschny Borges 1 \\ ${ }^{1}$ Universidade do Estado de Santa Catarina (UDESC) \\ Florianópolis - SC - Brazil \\ martha.borgesdudesc.br
}

\begin{abstract}
The paper presents the implementation of the Program International E-Cultures in Brazil. This is a project developed by the research team IDEOUniversity of Jaén whose goal is to promote exchanges between students from Europe and Latin America, the 6th grade of elementary school with a view to the development of intercultural education mediated by technology. The case study was conducted through observations and questionnaires with students in Brazil, a public school. The results showed that learning another culture first requires knowledge and appreciation of their own culture. The synchronous activities were most effective for the emergence of interaction between students.
\end{abstract}

Resumo. $O$ artigo apresenta o processo de implementação da Rede Internacional E-Culturas no Brasil. Trata-se de um projeto desenvolvido pela equipe de pesquisa IDEO - da Universidad de Jaén cujo objetivo é promover o intercâmbio entre alunos europeus e latino-americanos, do $6^{\circ}$ ano do Ensino Fundamental com vistas ao desenvolvimento da educação intercultural mediada pela tecnologia. $O$ estudo de caso foi realizado por meio de observações e questionários junto aos estudantes brasileiros, de uma escola pública. Os resultados mostram que a aprendizagem de outra cultura requer, em primeiro lugar, o conhecimento e valorização de sua própria cultura. Além disto, as atividades síncronas foram as mais eficazes para a emergência da interação entre os estudantes.

\section{Introdução}

Na sociedade contemporânea, testemunhamos uma pluralidade de realidades sociais, cada uma com dimensões próprias em termos culturais, étnicos, comunicativos, religiosos, etc, que por sua vez demandam ações específicas. Segundo Falteri (1998) os indivíduos não são mais portadores de uma maneira única de ver o mundo, de tomar decisões, de agir e, portanto, de organizar suas experiências de vida e atribuir-lhes um significado. Os sujeitos estabelecem diversas e híbridas relações e é neste cenário atual que somos convidados a resignificar nossos conceitos, nossas atitudes e nossos valores.

Este quadro se acentua com o processo de globalização, um fenômeno inevitável. Mas a direção na qual ele pode se desenvolver não é necessariamente predeterminado. Estamos diante de um modelo particular de globalização, que tem um 
forte componente econômico e que, portanto, promove um processo de exclusão. Este modelo se apoia nos critérios de hegemonia em função dos interesses dos grandes grupos econômicos [Gentili, 2004]. Entretanto, o processo de globalização pode ter outro modelo, que considere as dimensões socioculturais, políticas, linguísticas e educacionais.

A diversidade cultural está presente nas sociedades e ela forma e constitui os sujeitos. Assim, a cultura é o sujeito e o sujeito é cultura. A cultura orienta as ações, as atitudes e as práticas sociais de uma sociedade [Bruner, 2008]. Quando as culturas se relacionam, isso não significa que tenha havido uma "contaminação" cultural, no sentido de uma cultura extinguir outra. Pelo contrário, as relações entre as diferentes culturas podem, além de preservar a uma cultura, enriquecer as outras.

Educadores envolvidos em um processo de globalização inclusiva, articulada com a diversidade cultural, buscam formas de estabelecer novas abordagens que vão além dos limites da cultura local, promovendo o acesso e a valorização de outras culturas.

Hoje, com a expansão das tecnologias da informação e comunicação - TIC, é possível a promoção da interação entre diferentes sujeitos, por meio dos ambientes virtuais de aprendizagem (AVA). Muitos estudos sobre os usos da tecnologia foram realizados, no quadro da "sociologia dos usos" [Alava, 2002] ou da "história social das tecnologias" [Castells, 2003]. Segundo Millerand (1998; 1999) e Michel de Certeau (1990) estamos em uma fase chamada de apropriação de tecnologia.

Nesta abordagem, os estudos se fundamentam no uso real das tecnologias, nas práticas que os sujeitos concretizam ao se apropriarem das tecnologias. Estes estudos buscam investigar a diferença entre os usos ideais das tecnologias (abordagem da inovação) e os usos prescritos (abordagem da difusão). Os estudos de Perriault (1989) mostram que os usuários apresentam “...diferenças, variações, 'armadilhas' no uso das tecnologias" [Perriault, 1989, p. 14]. Estas variações são chamadas por Rabardel de "catacrese" [Rabardel, 1995, p. 124] e compõem o processo de gênese instrumental [Rabardel, 1995, 2004].

As tecnologias digitais mudam ainda o processo de criação, transmissão, codificação, acesso e armazenamento do conhecimento de uma forma nunca vista antes, incorporando num mesmo espaço multifacetado, o ciberespaço, todas as outras tecnologias intelectuais [Lévy, 1995, 2000, Moran, Masetto et Beherens, 2003). Neste sentido, Borges (2007) analisa que o ciberespaço pode se constituir em um lugar para expressar diferenças e para a colaboração mútua entre sujeitos que partilham seus conhecimentos. Este tipo de relação se estabelece a partir de uma intervenção educativa intencional, que promova processos de reelaboração de sentido construído pelos diferentes grupos culturais que compõem esta relação.

Assim, considerando os eixos apresentados: a possibilidade de promoção da globalização inclusiva, a valorização e o respeito à diversidade cultural como um dos elementos constitutivos dos sujeitos e o processo de apropriação de tecnologias digitais, a equipe de pesquisa da Universidade de Jaen, Espanha, desenvolveu um projeto intitulado Rede Internacional E-Culturas. O principal objetivo desta Rede é promover o desenvolvimento da educação intercultural 
As questões que este estudo pretende responder são: quais são as contribuições que a Rede E-Culturas traz para a emergência de uma educação intercultural, por meio de um ambiente de aprendizagem virtual que promove o intercâmbio entre estudantes de diferentes culturas? As atividades desenvolvidas promovem a compreensão, a valorização e o respeito a outras culturas pelos estudantes brasileiros?

Para respondermos a estas questões, inicialmente apresentamos uma breve revisão conceitual, de apresentação do conceito de interculturalidade e sua relação com o conceito de multiculturalismo.

\section{Da Multiculturalidade à Interculturalidade}

Embora os conceitos de multiculturalismo e de interculturalismo possam ser considerados sinônimos por alguns autores, eles não o são, nem em termos etimológicos, nem em termos práticos.

O prefixo "multi" significa muitos, já o "inter", significa relação entre duas ou mais partes. Multiculturalismo é, então, um termo de origem anglo-saxônica, enquanto que interculturalismo é um termo mais usado na Europa. Para se compreender a relação entre os dois termos é necessário considerar que uma mudança do multiculturalismo em direção à interculturalidade se constitui em um caminho a ser percorrido. $O$ multiculturalismo considera a simples coexistência de diferentes culturas. Por outro lado, a interculturalidade significa a plena integração de diversas culturas, que dá origem a uma outra cultura, ou seja, uma cultura híbrida no sentido proposto por Michel de Certeau (1990).

A sociedade é multicultural uma vez que coexistem duas ou mais culturas num mesmo espaço. No entanto, a coexistência não implica necessariamente convivência. Assim, a educação será sempre intercultural ou não será educação, como afirma Padilla (2007, p.47). Na sociedade atual não podemos apenas solicitar aos educadores que sensibilizem, incentivem seus alunos a respeitarem outras culturas. $\mathrm{O}$ respeito é o pilar para a emergência de um trabalho pedagógico. Mas para se desenvolver esses valores é preciso praticá-los.

Segundo Essomba (2008, p.24) o multiculturalismo é um modelo específico de gestão da diversidade cultural de influência anglo-saxônica que pressupõe a liberdade de cada cidadão para desenvolver seus costumes culturais como quiser, de falar a língua da sua escolha e de professar a religião de acordo com suas crenças, desde que o exercício destas liberdades não represente qualquer afronta à liberdade de outros cidadãos.

Entretanto, para este mesmo autor, a interculturalidade é também um modelo de gestão da diversidade cultural. Mas ela difere em essência, em localização e em sentido da multiculturalidade. A interculturalidade nasceu na França na década de setenta com a intenção de dar respostas à realidade da imigração. Assim, a interculturalidade significa o respeito à igualdade de todos, seja perante a lei, seja nas relações cotidianas. " $A$ interculturalidade pressupoe um processo de empatia, não apenas para conhecer os outros, mas para compreendê-los e a partir daí estabelecer os meios reais de comunicação" [Essoba, 2008, p.25]. Ou seja, ela promove trocas culturais em um espaço dinâmico e criativo, que enriquece a convivência cotidiana. 
De acordo com as informações e a sensibilização dos cidadãos, a diversidade cultural pode promover uma sociedade apenas multicultural ou pode promover uma verdadeira cidadania intercultural, onde a convivência transcende à coexistência. E neste caso, a educação se constitui em importante ferramenta.

\section{A Rede Internacional E-Culturas}

A Rede E-Culturas foi idealizada e é mantida pelo Grupo de Investigación IDEO da Universidade de Jaén - Espanha. Iniciada efetivamente no decorrer do ano de 2005, conta atualmente com a participação de mais de 500 estudantes e professores e pesquisadores de sete países: Espanha, Brasil, Argentina, Portugal, Reino Unido, Chile e Paraguai.

O propósito da Rede é possibilitar a emergência de uma educação intercultural. Para isso busca favorecer a interação e o conhecimento cultural entre estudantes e professores de centros educativos da Província de Jaén (Espanha) e dos países envolvidos, utilizando um ambiente virtual desenvolvido para este fim. Este ambiente oferece ferramentas de comunicação síncronas e assíncronas, como fórum, chat, correio eletrônico, além das atividades de cooperação propostas aos estudantes (wwww.eculturas.org).

Com o objetivo de participarmos da Rede E-Culturas, realizamos uma parceria entre a Universidad de Jaén, a Universidade do Estado de Santa Catarina - UDESC e a Secretaria de Estado de Educação. A seguir. Definimos a escola de ensino fundamental para participar da experiência. A escola definida se localiza na cidade de Florianópolis e os estudantes que participaram das atividades de intercâmbio compõem uma turma de $6^{\text {a }}$ série. O mesmo ocorreu com as escolas espanholas e argentinas. No Brasil, os estudantes participaram de maneira espontânea das atividades, no contra-turno das aulas e de forma espontânea.

\section{Atividades Realizadas em Escola Pública de Ensino Fundamental}

Este artigo apresenta os resultados da investigação realizada junto aos estudantes da $6^{\mathrm{a}}$ serie da escola brasileira realizada no período de 2010/2011. A pesquisa se caracteriza como estudo de caso e foi realizada junto a quatorze estudantes, com idades entre doze e quatorze anos.

Os instrumentos de coleta de dados foram as observações comportamentais dos estudantes na realização das atividades propostas e do professor que conduziu as ações pedagógicas. O objetivo das observações foi de acompanhar e registrar todas as ações realizadas e de identificar os tipos de interações que estes sujeitos estabeleceram entre eles e seus colegas de equipe de outros países. No final de cada etapa das atividades, aplicamos um questionário com o objetivo de coletar as impressões dos estudantes e do professor, sobre as atividades mais interessantes, as mais difíceis, as sugestões para o aprimoramento da Rede e especialmente sobre o que eles aprenderam de outras culturas, em termos de semelhanças e de diferenças com a cultura deles.

Inicialmente os estudantes receberam logins e senhas individuais para acessarem o ambiente virtual e iniciarem a postagem de suas informações e apresentações pessoais aos estudantes da Espanha e da Argentina. A seguir o próprio sistema agrupou os 
estudantes em equipes de maneira aleatória, de acordo com os seguintes critérios: equipes de nacionalidades diferentes e equilíbrio no número de meninos e meninas. Cada equipe foi composta por quatro estudantes sendo dois brasileiros (de cidades diferentes), um espanhol e um argentino.

A Rede propõe três etapas de atividades previamente organizadas: Meu Álbum/Nosso Álbum, Mosaico composto por quatro módulos interculturais e Jogo Interativo. Na primeira, os estudantes em equipes com as três nacionalidades foram desafiados a construírem um álbum de apresentação pessoal e interativo, onde eles realizaram atividades escritas e disponibilizaram fotos (de cada estudante, de suas famílias, de suas escolas, de seus professores, de seus amigos). Os objetivos desta fase foram: familiarizar os estudantes com o ambiente virtual, estabelecer os primeiros contatos entre eles e as outras culturas, reconhecer suas identidades pessoais em relação aos outros estudantes.

Assim, cada estudante ao se apresentar e postar sua foto iniciou um diálogo com os colegas de equipe. Esta etapa motivou significativamente os estudantes, eles manifestaram muito interesse em conhecer seus colegas de equipe, como eles eram, o que eles faziam, como eram suas cidades, etc. A seguir eles apresentaram suas famílias, suas escolas e suas cidades. Junto a estas apresentações, eles também postaram fotos, explicaram os costumes de sua cultura, os monumentos e locais característicos.

Esta atividade causou certa angústia e alvoroço nos estudantes brasileiros, uma vez que eles deveriam enumerar e apresentar os locais mais característicos de suas cidades, deveriam narrar um conto ou uma atividade folclórica, além de descreverem uma festa local. Nestes momentos, os estudantes que se encontravam na mesma sala informatizada, dialogando com os estudantes de outras nacionalidades entravam em desacordo na escolha dos aspectos a serem apresentados.

No momento de apresentar os principais monumentos e locais de Florianópolis, os próprios estudantes perceberam que o conhecimento que eles tinham de sua cidade era restrito. Foi identificado que um número significativo deles (6 sobre 14) não haviam nascido na cidade. Eles fazem parte de famílias que vieram de outros estados brasileiros ou de outras cidades de Santa Catarina, para Florianópolis, com o objetivo de encontrarem trabalho. Na verdade este fato exemplifica as estatísticas de migração que a cidade apresenta desde os anos oitenta [IBGE, 2010].

Portanto, para apresentarem sua cidade, os estudantes tiveram primeiro, que pesquisarem sobre ela. A maioria deles nunca havia visitado locais turísticos que ficam longe do bairro da escola. Assim, o projeto organizou alguns passeios ao centro da cidade para eles conhecerem estes locais, além de algumas praias que tem forte apelo turístico, mas eram desconhecidas deles. Neste passeio eles realizaram várias anotações e fotos dos locais para depois apresentarem aos colegas espanhóis e argentinos. Remarcamos ainda, que as descrições que eles elaboraram foram bastante realistas, eles descreveram os aspectos positivos dos locais (como as belas praias e monumentos), mas também os negativos (como a difícil mobilidade urbana, a violência e a falta de espaços culturais e esportivos). Estes dados mostram que os objetivos iniciais do projeto foram atendidos, no sentido que, para se conhecer e respeitar outras culturas, é preciso primeiro, conhecer e valorizar sua própria cultura. 
Outro aspecto a destacar se refere à questão da língua. Percebemos que os estudantes brasileiros, desde o inicio das atividades, tentavam escrever aos seus colegas na língua deles, mesmo sem nunca terem aprendido o espanhol. Embora o ambiente tivesse a opção de tradução e todas as atividades estavam disponíveis nas duas línguas, eles queriam se comunicar em espanhol. Assim, de forma espontânea, eles se apropriaram da língua espanhola, das expressões cotidianas. Já os estudantes espanhóis e argentinos não demonstraram este tipo de iniciativa. Este fato pode demonstrar que os estudantes brasileiros são abertos a outras culturas e tentam se comunicar e se fazer entender por outras nacionalidades. Este fenômeno pode estar relacionado à própria constituição do povo brasileiro, que passou por um processo de forte imigração e também ao processo de migração de suas famílias para Florianópolis.

Nesta fase, os estudantes perceberam outras diferenças entre as três culturas, especialmente relacionadas ao acesso e ao uso das tecnologias digitais. Para a realização das fotos, a maioria dos estudantes brasileiros não tinha um máquina digital em casa. Foi necessário 'emprestarmos' uma para eles, cada um a seu turno, para que eles pudessem fazer as fotos da família e do seu bairro. Este fato não foi imperceptível para os estudantes.

Outra dificuldade explicitada por meio dos questionários foi com relação à busca de imagens da sua cidade e especialmente do seu bairro, na Internet. Eles constataram que mesmo em sites de referência em busca de imagens, como Google Imagens e Google Earth, estas fotos são em número bem reduzido se comparadas com as fotos das cidades espanholas. Além das imagens de sua cidade serem de qualidade ser inferior, eram bastante desatualizadas.

As diferenças em termos de estrutura familiar também foram remarcadas. Eles perceberam que, ao contrário dos estudantes espanhóis, todas suas mães trabalhavam fora de casa. Por outro lado, apenas três, das sete mães espanhola,s trabalhavam fora de casa. Este fato oportunizou um acalorado debate entre os estudantes brasileiros, porque a maioria deles imaginava que as mulheres europeias exerciam alguma profissão. 


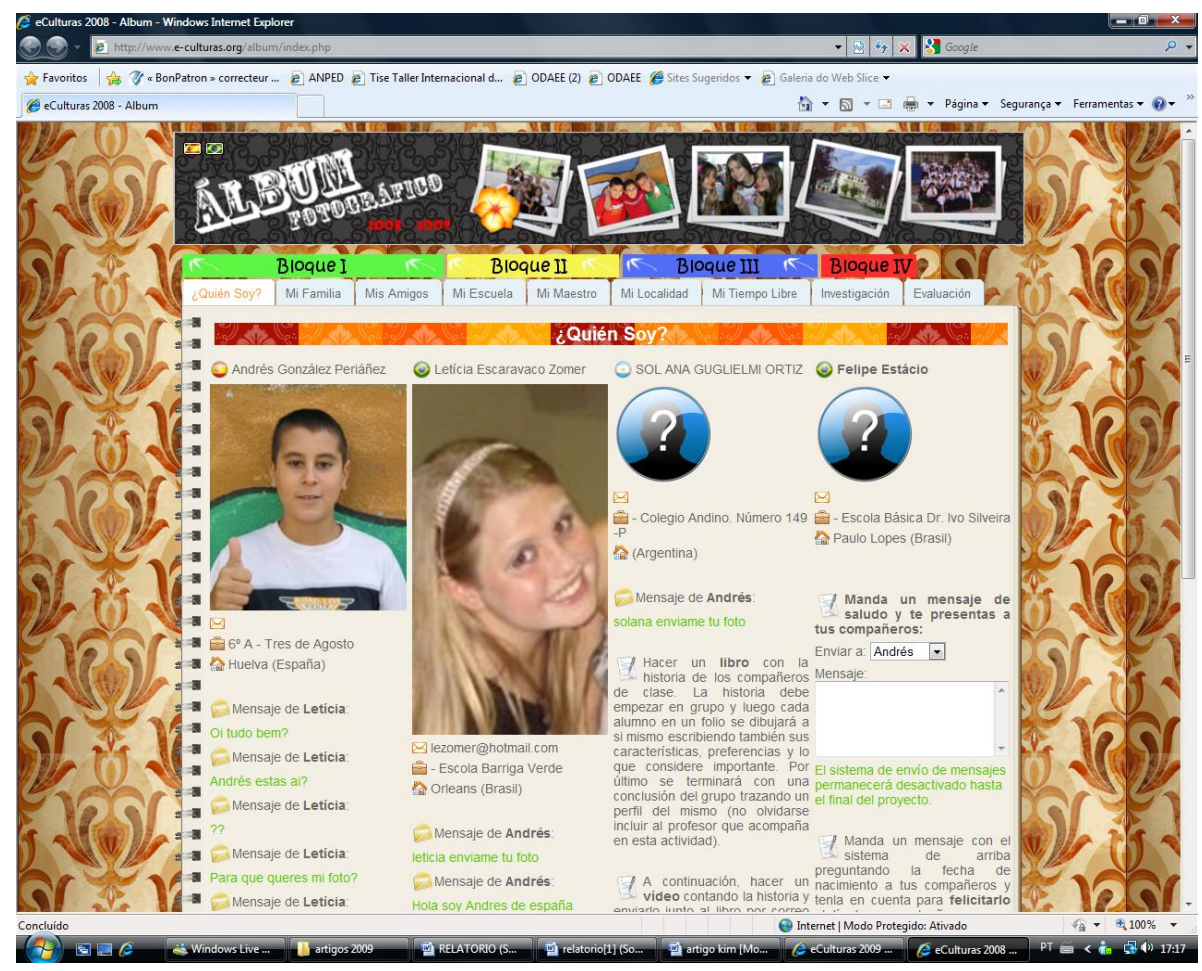

Figura 1. Página da fase "Meu Álbum/Nosso Álbum"

A segunda etapa do projeto denominada de Mosaico é uma metáfora que se propõe a trabalhar com o conjunto de diferenças que unem e separam as diferentes culturas, mas que favorecem uma grande riqueza cultural. Saber integrar a cultura como um aspecto natural, que faz parte do nosso desenvolvimento como indivíduos, é o principal objetivo desta fase. É como um pedaço de mosaico cujas diretrizes são as mesmas para todos: respeito, igualdade, liberdade, solidariedade e democracia. O tema principal da fase é "O continente americano e o continente europeu: um mundo para todos". As atividades propostas foram de pesquisa, de elaboração de textos escritos, de postagem de imagens e de áudios produzidos pelos alunos ou disponíveis na web.

Durante a realização desta fase, os estudantes foram agrupados em equipes de mesma nacionalidade, compostas por dois ou três sujeitos. A estrutura desta fase é fundamentada na metodologia de projetos, que se centra na identificação de um problema que deve ser resolvido pelos alunos. Ela estimula a pesquisa como forma de buscar uma solução, permitindo aos estudantes a aplicação de seus conhecimentos como ferramenta para resolver o problema ou sugerir melhorias. Este tipo de aprendizagem se orienta em direção à ação [Hernandez, 1998]. No E-Culturas, as equipes foram desafiadas a elaborarem um "guia turístico" de seu país, apresentando os aspectos geográficos, culturais e sociais mais importantes de cada país.

À medida que os estudantes elaboravam o seu "guia turístico", eles foram conhecendo seu próprio país, os principais rios, sua costa, flora, fauna, as diferentes regiões brasileiras, sua vegetação, os pontos turísticos, a economia. Nos questionários os estudantes realizaram afirmações como a seguinte: "Eu não sabia que o rio Amazonas se origina no Peru, e não o Brasil, nem que é o maior rio do mundo ... uauuuu!". Ou ainda: "Eu não sabia que o Cristo Redentor no Rio de Janeiro tem 38 metros de altura e foi um presente da França para o Brasil”". 
Outro fato que nos surpreendeu foi que eles previlegiaram, em seus "guias turísticos", as descrições sobre aspectos culturais da cidade de Florianópolis em detrimento de descrições sobre o Brasil. Eles mostraram que se sentiam mais à vontade descrevendo sua cidade que seu país. Segundo Gilberto Ferreira da Silva (s/d), existe sempre uma relação aproximação e de distanciamento da cultura local na relação entre dois universos: o nacional e o regional (local). Esse movimento é captado tanto pela visão expressa pelos estudantes quanto pelos professores. "O 'brasileiro' é o outro, o estranho, o distante que não faz parte de meu espaço e de minhas relações. Posso falar desse outro sem pruridos, fazer críticas, acusar e no minar - o "brasileiro" é lento, safado, preguiçoso" [Silva, p.6]. Por outro lado, quando a referência é local, estas características assumem outros sentidos, outras representações. Ou seja, um discurso que exclui e inclui.

Neste sentido, pudemos verificar que os alunos apresentaram maior dificuldade para realizarem as atividades que abordavam aspectos internacionais, que solicitavam o reconhecimento de monumentos como a cidade de Machu Pichu, o Coliseu, a Estátua da Liberdade, a Torre Eiffel, Ilha de Páscoa, etc. Assim, constatamos que o conhecimento sobre a cultura internacional é ainda mais reduzido do que o de sua cultura local.

A última fase do projeto denominada de "Quijotin parte em busca do tesouro" promove a realização de um jogo interativo onde as equipes de estudantes dos três países devem elaborar um roteiro de viagem para o personagem "Quijotin", o filho de Dom Quijote e de Dulcinéia. Ele irá visitar a região da Andaluzia, viajar até o Brasil e ir para a Argentina. Trata-se de um jogo colaborativo onde não existem vencedores. Os objetivos foram: realizar projetos compartilhados, tomar consciência da existência de uma cultura comum e adquiri uma identidade pessoal e do grupo.

O jogo apresentou uma série de seis itinerários, onde cada membro da equipe deveria percorrer e ao mesmo tempo, responder a seis perguntas que abordavam vários aspectos da cultura das regiões da viagem. Se os alunos respondessem às perguntas corretamente, eles se moviam para as próximas etapas, se não, eles deveriam buscar o conhecimento para responder corretamente. No final, no último itinerário, todos receberam um certificado nominal, com os nomes de suas escolas e de seus respectivos países: o tesouro.

O jogo motivou muito os estudantes, pois eles puderam interagir novamente com os estudantes de outras nacionalidades. Eles perceberam que as dúvidas e dificuldades que encontraram na realização do jogo, foram as mesmas para todos. A diferença foi que nas outras escolas, os professores dos conteúdos curriculares, como geografia, história, ciências e línguas, participaram de forma intensa, o que não foi o caso na escola brasileira.

Outro aspecto identificado na investigação é que estudantes aprenderam a fazer pesquisas na Internet de forma mais produtiva, eles aprenderam a selecionar a informação, a críticá-la e a serem independentes, como afirma um estudante: "No E-Culturas fizemos várias pesquisas em sites, indicados na plataforma por links. Então eu usei os mesmos sites de pesquisa para completar o trabalho de outros professores da Escola. Foi legal!’”. 
Para Maria Isabel Orofino (2002) a escola tem um papel importante como mediadora da informação veiculada pelos midias. É um espaço para o uso e a apropriação das tecnologias de comunicação em termos de acesso, de reelaboração da informação e de produção de conhecimento.

\section{Considerações Finais}

De modo geral, tanto os estudantes como o professor avaliaram de maneira positiva suas participações na Rede E-Culturas. Além disto, algumas repercussões no cotidiano escolar puderam ser detectadas e relacionadas à participação na Rede. Dentre elas podemos destacar: o processo de engajamento dos estudantes na realização das atividades, especialmente aquelas de apresentação de si, de sua família, de sua escola e classe foi significativo. Esta etapa de apresentação, que 'deixa livre' os alunos para interagirem espontaneamente com os colegas de outras nacionalidades, em equipe pequenas, foi fundamental para o desenvolvimento da curiosidade e do conhecimento de outras realidades e culturas.

Percebemos a dificuldade dos estudantes nas atividades que envolviam uma apresentação de sua cidade e de sua cultura, especialmente por que estes estudantes não conhecem a cultura nacional. Esta dificuldade foi acentuada pelo pouco comprometimento por parte dos professores, que, apesar das reuniões iniciais, não se engajaram no projeto. As atividades foram realizadas não por professores do currículo regular, mas pela coordenadora da sala informatizada, que não tinha nenhum 'compromisso' com o ensino e a aprendizagem de uma determinada disciplina e, assim, não poderia integrar as atividades do projeto com as atividades curriculares de ensino. Fica o questionamento, esta falta de envolvimento se deu em função de pouca familiaridade com o uso das tecnologias ou por comodismo, por não haver uma vontade de modificar uma prática docente já cristalizada?

Finalizando podemos dizer que os alunos modificaram suas concepções sobre diferentes culturas, demonstraram, por meio de reflexões e questionamentos, que sua visão de mundo e especialmente da sua cultura local, próxima a eles se tornou mais consistente e critica.

\section{Referências}

Alava, Séraphin (org) (2002) “Ciberespaço e formações abertas”. Rumo a novas práticas educacionais? Porto Alegre: Artmed.

Borges, Martha K. (2007). "Educação e cibercultura": perspectivas para a emergência de novos paradigmas educacionais. In Vallejo, A. Pantoja, Zwierewicz, Marlene (org). Sociedade da informação, educação digital e inclusão p. 53-86. Florianópolis, Insular.

Bruner, Jérôme. (2008). “Culture et modes de pensé, l'esprit humain dans ses œuvres”. Paris, Retz.

Castells, M. (2003). “A galáxia da Internet”. Reflexões sobre a Internet, os negócios e a sociedade. Rio de Janeiro: Zahar.

De Certeau (1990). “L'invention du quotidien”. Paris : Union Générale d’Éditions. 
Falteri, Paola. (1998). "Interculturalismo e culturas no plural". In Fleuri, Reinaldo M (org) Intercultura e movimentos sociais. p. 112-132. Florianópolis, Mover.

Gentilli, Pablo (2004). “A globalização libertadora tem de sustentar um conjunto de valores” Revista Pátio, ano VII, n 28, p. 26-40.

Hernandez, Fernando \& Ventura, Montserrat. (1998). "A organização do Currículo por Projetos de trabalho". $5^{\text {a }}$ ed. Porto Alegre, Artes Médicas.

IBGE. Instituto Brasileiro de Geografia e Estatística. (2010). "Censo demográfico". Brasília, MEC. Disponível em http://www.ibge.gov.br/home/estatistica/populacao/censo2000/migracao/tabela_regio es.shtm. Acesso em fev 2010.

IBGE. Instituto Brasileiro de Geografia e Estatística. (2010), Brasil: 500 anos de povoamento. Brasilia, MEC. Disponivel em http://www.ibge.gov.br/brasil500/index.html Acesso em fev 2010.

Lévy, Pierre. (1995). "As tecnologias da inteligência": o futuro do pensamento na era da informática. Rio de Janeiro: Ed 34, 2a edição.

Lévy, P. (2000). “Cyberculture”. Paris, La Découverte.

Millerand, F. (1998). "Usages des NTIC": les approches de la diffusion, de l'innovation et de l'appropriation $\left(1^{\mathrm{a}}\right.$ parte). Disponível em : http://commposite.uqam.ca/98.1/articles/ntic 1.htm. Acesso em fev 2009.

Millerand, F. (1999). "Usages des NTIC": les approches de la diffusion, de l'innovation et de l'appropriation ( $2^{\mathrm{a}}$ parte $)$ Disponível em : http://commposite.uqam.ca/99.1/articles/ntic 1.htm. Acesso em fev 2009.

Moran, J. M., Masetto, M. T. and Beherens, M. A. (2003) "Novas tecnologias e mediação pedagógica". Campinas, SP: Papirus.

Orofino, Maria Isabel. (2002). "Mídia e educação": contribuições dos estudos da mídia e comunicação para uma pedagogia de meios na escola In: Fleuri, Reinaldo Matias (Org.). Educação Intercultural: mediações necessárias. Florianópolis: EDUFSC.

Perriault, J. (1989). "La logique de l'usage”. Essai sur les machines à communiquer. Paris: Flammarion.

Rabardel, P. (1995). "Les hommes et les technologies". Approche cognitive des instruments contemporains. Paris: Armand Colin.

Rabardel, P. (2004). "Hommes, artefacts, activités": perspective instrumentale. In Falzon, Pierre. Ergonomie. p. 251-268. Paris, Presses Universitaire de France.

Silva, Gilberto Ferreira (s/d). "Interculturalidade e Educação de Jovens": processos identitários no espaço urbano popular. Disponível em: http://www.lppuerj.net/olped/documentos/0025.pdf. Acesso nov. 2008. 